\title{
High Range Resolution Profile Construction Exploiting Modified Fractional Fourier Transformation
}

\author{
Feng Wang, Defu Jiang, and Huawei Chen \\ College of Computer and Information, Hohai University, Focheng West Road, No. 8, Jiangning District, Nanjing, Jiangsu 211100, China
}

Correspondence should be addressed to Feng Wang; jihonghope@aliyun.com

Received 3 July 2015; Accepted 29 November 2015

Academic Editor: Aime’ Lay-Ekuakille

Copyright (C) 2015 Feng Wang et al. This is an open access article distributed under the Creative Commons Attribution License, which permits unrestricted use, distribution, and reproduction in any medium, provided the original work is properly cited.

This paper addresses the discrimination of closely spaced high speed group targets with radar transmitting linear frequency modulation (LFM) pulses. The high speed target motion leads to range migration and target dispersion and thereby the discriminating capability of the high range resolution profile (HRRP) deteriorating significantly. An effective processing approach composed of stretch processing (SP), modified fractional Fourier transform (FrFT), and multiple signal classification (MUSIC) algorithm is proposed to deal with this problem. Firstly, SP is adopted to transform the received LFM with Doppler distortions into narrow band LFM signals. Secondly, based on the two-dimensional range/velocity plane constructed by the modified FrFT, the velocity of the high speed group target is estimated and compensated with just one single pulse. After the compensation of range migration and target dispersion simultaneously, the resolution of the HRRP achieved by single pulse transmission improves significantly in the high speed group targets scenarios. Finally, MUSIC algorithm with superresolution capability is utilized to make a more explicit discrimination between the scatterers in comparison with the conventional SP method. Simulation results show the effectiveness of the proposed scheme.

\section{Introduction}

The task of detecting unresolvable group targets raises new challenges for both target detection and discrimination. A group target refers to a group of subtargets in a formation flying in the same direction with the same speed, such as aircrafts in a close formation, missiles with multiple warheads, or space debris in outer space orbit [1-4]. High range resolution profile (HRRP) of a wide band radar is an effective technique for the discrimination of a group target, and it has been intensively investigated in the field of automatic target recognition (ATR) [5-7]. The resolution of group targets can be improved by the increase of the system bandwidth as well as the exploitation of superresolution algorithms in range dimension. The increase of the bandwidth means more difficulties in the manufacture of transmitting tube and receiver end. On the other hand, superresolution algorithm is an alternative way to get range profiles with improved resolution beyond what is limited by the bandwidth of the signal [8-10]. Several suggestions for dealing with the superresolved range profiles can be found in open literatures.
A method to extract the range location of point scatters using a Prony model is investigated in [11]. Several key components to the algorithm, such as the removal of spurious scattering centers, are analyzed. Burg's AR method is discussed in [12] to generate high-resolution profiles. The poles of the HRRP are extracted using the method of AR modified covariance in [13]. Superresolution called multiple signal classification (MUSIC) is investigated in [14] to form the prominent peaks in range-Doppler dimension. The performance of MUSIC algorithm is further investigated in [15] to generate superresolved range profiles. Relaxation-based algorithms with superresolution capability are proposed in [16] for HRRP feature extraction of moving targets consisting of scatterers closely spaced in range. Superresolution algorithms, including adaptive pulse compression algorithm and least-squares estimation, are investigated in [17] in comparison with the nominal resolution of the standard matched filter. Diagonal loading of the two superresolution approaches is discussed in the case of matrix ill-conditioning. Linear predictive bandwidth extrapolation techniques are used in [18] for obtaining improved range superresolution. Side-lobe 
cancellation and singular value decomposition are utilized in computing the linear prediction filter coefficients.

Though HRRP with superresolution has been achieved by the above-mentioned methods, range profile distortion caused by target motions is not taken into consideration in the above literatures. The radial velocity of the target leads to range migration and dispersion of the HRRP $[19,20]$. Hence, the characteristics extracted from the HRRP reduce greatly. Several techniques, such as target velocity compensation using maximum likelihood (ML) estimation [21], phase coded stepped-frequency waveform transmission [22, 23], and two successive stepped-frequency pulse train transmission [24], have been proposed to reconstruct the distorted HRRP. However, these techniques are based on the pulse train processing. For radar working in single pulse mode, little has been done in regard to range resolution in high speed target motion scenarios.

Pulse train transmission is essential to improve the resolution of velocity of a moving target. However, the HRRP based on single pulse is more efficient for the identification of a group target. To a conventional aerodynamic target, distortion caused by the target motion is not so severe during the pulse width of a single pulse. For high speed group target with multiple subtargets, such as a missile with multiple warheads or space debris flying in orbits, the Doppler effect leads to serious distortion on the HRRP.

The aim of this research is to design a methodology with further capability for feature extraction in case of severe Doppler distortion. A processing chain based on stretch processing (SP) [25-27], modified fractional Fourier transform (FrFT) $[25,26]$, and superresolution algorithm (such as MUSIC [28]) is proposed to make discrimination between the subtargets. For radar system exploiting linear frequency modulation (LFM) signal, SP is another efficient approach to realize the matched filter by looking for the echoes within a predefined range interval of interest [29-31]. SP is usually used in the wide band processing in synthetic aperture radar (SAR), reducing the bandwidth requirement of the subsequent processing. We choose the classical MUSIC algorithm as a tool to achieve higher range resolution profiles. Better discrimination results can be achieved by the utilization of superresolution algorithm in comparison with the conventional Fourier transform (FT) method.

Since the SP and MUSIC algorithms have been used for resolution in range dimension, the main difference of this paper from the open literatures lies in the utilization of FrFT. FrFT has become one of the most important tools applied in the field of radar signal processing [29]. By exploiting FrFT with a proper rotation factor, a chirp signal becomes a signal with single frequency in an alternative mapping $\mathrm{U}$ domain. The energy-focusing characteristic of the FrFT makes it a powerful tool in the field of matched filtering, target detection, and parameter estimation for radar systems utilizing chirp signals [32, 33]. FrFT algorithm is utilized in this paper to compensate for the distortion caused by Doppler effect for transmitted signals in LFM form. The unwanted dispersion of the HRRP caused by target motion can be easily removed by FrFT, providing focused HRRP with no dispersion. However, the range migration caused by frequency shift remains uncompensated. Hence, a kind of modified FrFT is proposed based on a priori knowledge of the transmitted signal. The target dispersion and range migration both can be compensated with the modified FrFT; thereby superresolution range profile and radial velocity are obtained simultaneously.

The organization of this paper is as follows. In Section 2, the model of the HRRP is analyzed and the conventional processing chain based on SP to achieve the HRRP is discussed. The range migration and target dispersion effect caused by target motion are investigated in detail. In Section 3, the defects of Doppler distortion compensation using FrFT and the advantages of modified FrFT are investigated. The processing chain based on SP, modified FrFT, and MUSIC is proposed for LFM signal transmission scenarios in Section 4. In Section 5, the performance of the modified FrFT for Doppler compensation and velocity estimation is tested through numerical simulations. The performance comparison between the conventional and the proposed processing chain is also validated by computer simulations. Finally, Section 6 summarizes the work of this paper.

\section{Problem Formulation}

Group target is composed of a group of subtargets flying in close formation with the same speed. The HRRP achieved by a wide band radar can be recognized as the transfer function of the group target. The transfer function of the target echoes with different time delays can be expressed as $[1,2]$

$$
\begin{aligned}
& \mathbf{h}(t)=\left[\alpha_{0} \delta\left(t-\tau_{0}\right), \alpha_{1} \delta\left(t-\tau_{1}\right), \ldots, \alpha_{i} \delta\left(t-\tau_{i}\right), \ldots,\right. \\
& \left.\alpha_{N-1} \delta\left(t-\tau_{N-1}\right)\right]^{T}
\end{aligned}
$$

where $N$ denotes the number of the scatterers, superscript $[\cdot]^{T}$ transpose operation, $t$ the time variable, $\tau_{i}$ the time delay of the $i$ th subtarget, $\alpha_{i}$ the complex amplitude coefficient of the $i$ th subtarget, and $\delta(t)$ the impulse response. The received scatterers of a moving target can be modeled as

$$
\begin{aligned}
y(t) & =\exp \left\{j 2 \pi f_{d} t\right\} \cdot[\mathbf{h}(t) \otimes s(t)]+n(t) \\
& =\exp \left\{j 2 \pi f_{d} t\right\} \sum_{i=0}^{N-1} \alpha_{i} s\left(t-\tau_{i}\right)+n(t),
\end{aligned}
$$

where $\otimes$ represents the convolutional operator, $s(t)$ denotes the transmitted signal, $f_{d}$ is the Doppler shift of the group target, $\exp \{j[\cdot]\}$ is the exponential representation of complex numbers, and $n(t)$ is the addictive white Gaussian noise (AWGN) in the receiver channel.

For radar transmitting LFM signals, the transmitting LFM pulse can be written as

$$
\begin{aligned}
s(t) & =\exp \left\{-j 2 \pi\left(f_{c} t+0.5 \mu t^{2}\right)\right\} \\
& =\exp \left\{-j 2 \pi\left(f_{c}+0.5 \mu t\right) t\right\},
\end{aligned}
$$

where $\mu=B / T$ is the frequency modulation rate, $B$ the signal band width, $T$ the pulse width, and $f_{c}$ the carrier frequency 


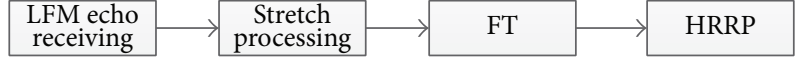

FIGURE 1: Conventional processing chain using the SP technique.

\begin{tabular}{|c|c|c|c|}
\hline $\begin{array}{l}\text { LFM echo } \\
\text { receiving }\end{array}$ & $\begin{array}{c}\text { Stretch } \\
\text { processing }\end{array}$ & MUSIC & HRRP \\
\hline
\end{tabular}

FIGURE 2: Processing chain using SP and MUSIC approach.

of the transmitted signal. According to (2), the scatterer of the $i$ th subtarget with Doppler shift $f_{d}$ can be expressed as

$$
\begin{aligned}
& x_{i}(t)=\alpha_{i} s_{i}\left(t-\tau_{i}\right) \exp \left\{j 2 \pi f_{d} t\right\}=\alpha_{i} \\
& \cdot \exp \left\{-j 2 \pi\left[f_{c}\left(t-\tau_{i}\right)+0.5 \mu\left(t-\tau_{i}\right)^{2}\right]\right\} \\
& \cdot \exp \left\{j 2 \pi f_{d} t\right\} .
\end{aligned}
$$

The group target with $N$ scatterers can be described by the following sum:

$$
\zeta(t)=\sum_{i=0}^{N-1} x_{i}(t)
$$

The reflections in the receiver end with channel noise can be written as

$$
y(t)=\zeta(t)+n(t) .
$$

The effect of noise is neglected in the following analysis for simplicity. Without considering the influence of noise, the superposition of the scatterers of the $N$ subtargets in the radar receiver is of the following form:

$$
\begin{aligned}
& y(t)=\zeta(t)=\exp \left\{j 2 \pi f_{d} t\right\} \\
& \quad \cdot \sum_{i=0}^{N-1} \alpha_{i} \exp \left\{-j 2 \pi\left[f_{c}\left(t-\tau_{i}\right)+0.5 \mu\left(t-\tau_{i}\right)^{2}\right]\right\} .
\end{aligned}
$$

2.1. SP for LFM Transmission. The conventional processing chain to achieve HRRP is presented in Figure $1[5,7]$. Reducing the sampling frequency greatly, the technique based on SP and FT has the same resolving performance as that of the pulse compression technique. Figure 2 shows an alternative processing chain based on superresolution in range dimension exploiting SP and MUSIC algorithms [14]. More features can be extracted by the superresolution algorithm in comparison with the conventional FT approach in Figure 1.

The LFM echoes after SP are converted into multiple frequencies if Doppler distortion caused by target motion is not taken into consideration. The SP technique is summarized as follows [25]:

(1) Designating the reference range and the characteristics of the LFM waveform.

(2) Exploiting SP for the receiving analog signal in the receiver during the interval of interest instead of the entire range span.
(3) Achieving the frequency spectrum of the signal by FT analysis.

(4) Converting each peak frequency into the corresponding range value using the frequency spectrum.

The reference signal for SP is of the form $[25,27]$

$$
u\left(t_{r}\right)=\exp \left\{-j 2 \pi\left(f_{c} t_{r}+0.5 \mu t_{r}^{2}\right)\right\},
$$

where $t_{r}$ is the signal window for SP defined in the range of

$$
t_{r} \in\left[\frac{2 R_{\min }}{c}, \frac{2 R_{\max }}{c}\right]
$$

where $c$ is the speed of light $\left(3 \times 10^{8} \mathrm{~m} / \mathrm{s}\right)$ and $R_{\max }$ and $R_{\min }$ represent the maximum and minimum range for processing, respectively. Correlating the reference signal in (8) with the received signal in (7), we have $[29,30]$

$$
\begin{aligned}
& z\left(t_{r}\right)=y\left(t_{r}\right) \cdot u^{*}\left(t_{r}\right)=\exp \left\{j 2 \pi f_{d} t_{r}\right\} \\
& \quad \cdot \sum_{i=0}^{N-1} \alpha_{i} \exp \left\{-j 2 \pi\left(-\mu \tau_{i} t_{r}-f_{c} \tau_{i}+0.5 \mu \tau_{i}^{2}\right)\right\},
\end{aligned}
$$

where $[\cdot]^{*}$ denotes complex conjugate. From formula (10), we can see that the time delay of the subtarget echoes has been converted into signals with different frequencies if the Doppler frequency $f_{d}$ is supposed to be constant. For conventional processing, FT is carried out after SP [27]:

$$
U(\omega)=\int_{t_{r}=2 R_{\min } / c}^{t_{r}=2 R_{\max } / c} z\left(t_{r}\right) e^{-j \omega t_{r}} d t_{r},
$$

where $\omega$ represents the angular frequency. It has been proven that SP has the same performance as that of the matched filtering technique. Target signatures extracted from the scatterers depend on the resolution of the spectrum of the FT, which is usually realized by fast Fourier transform (FFT). Therefore, the resolution improvement of the frequencies is of great benefit to the discrimination of the target.

2.2. Doppler Distortion on the HRRP. For group target in uniform motion, the Doppler frequency shift of the group target can be written as [19]

$$
f_{d}=\frac{2 v_{0}}{\lambda}=\frac{2 v_{0}}{c} f
$$

where $v_{0}$ denotes the radial velocity of the group target, $\lambda$ represents the wavelength of the transmitted signal, and $f$ is the instantaneous frequency. To a wide band signal, different $f_{d}$ is matched with different $\lambda$. According to formulas (3) and (12), we get the Doppler of a transmitted LFM signal:

$$
f_{d}=\frac{2 v_{0}}{c}\left(f_{c}+0.5 \mu t_{r}\right) .
$$


Hence, formula (10) can be rewritten as

$$
\begin{aligned}
& z\left(t_{r}\right)=\exp \left\{j 2 \pi \frac{2 v_{0}}{c}\left(f_{c}+0.5 \mu t_{r}\right) t_{r}\right\} \\
& . \sum_{i=0}^{N-1} \alpha_{i} \exp \left\{-j 2 \pi\left(-\mu \tau_{i} t_{r}-f_{c} \tau_{i}+0.5 \mu \tau_{i}^{2}\right)\right\} \\
& =\exp \left\{j 2 \pi \mu \frac{v_{0}}{c} t_{r}^{2}\right\} \cdot \exp \left\{j 4 \pi \frac{v_{0}}{c} f_{c} t_{r}\right\} \\
& \quad \cdot \sum_{i=0}^{N-1} \alpha_{i} \exp \left\{-j 2 \pi\left(-\mu \tau_{i} t_{r}-f_{c} \tau_{i}+0.5 \mu \tau_{i}^{2}\right)\right\} .
\end{aligned}
$$

From formulas (13) and (14), we can see that the variation of the Doppler frequency is proportional to the instant frequency of the transmitted LFM signal. The first exponential term in (14) corresponds to the frequency change of the signal due to target motion. The remaining signal after SP is still a LFM component due to the Doppler effect on the transmitted wide band LFM signals. From (14), the two components of Doppler can be represented as

$$
\begin{aligned}
& f_{d 1}=\mu \frac{v_{0}}{c} t_{r}=\mu \frac{B v_{0}}{T c} t_{r}, \\
& f_{d 2}=\frac{2 v_{0}}{c} f_{c} .
\end{aligned}
$$

The signal window of SP is defined to be equivalent to the pulse width of the transmitted signal:

$$
\frac{R_{\max }-R_{\min }}{c}=T \text {. }
$$

We get the frequency difference caused by component $f_{d 1}$ during the signal window:

$$
\Delta f=2 \frac{B v_{0}}{T c} \frac{R_{\max }-R_{\min }}{c}=2 \frac{B v_{0}}{c} .
$$

Note that the Doppler dispersion in (18) is proportional to the system bandwidth and the radial velocity of the group target. We can conclude that the first Doppler component $f_{d 1}$ results in the time variant characteristics of the Doppler frequency, which leads to undesirable target dispersion in the HRRP.

According to (14), the superposition of the single tones consisting of Doppler component $f_{d 2}$ in (16) and time delay components can be represented as

$$
\begin{aligned}
& g\left(t_{r}\right)=\sum_{i=0}^{N-1} \alpha_{i} \\
& \cdot \exp \left\{-j 2 \pi\left[\left(-\mu \tau_{i}-\frac{2 v_{0}}{c} f_{c}\right) t_{r}-f_{c} \tau_{i}+0.5 \mu \tau_{i}^{2}\right]\right\} .
\end{aligned}
$$

The time delay $\tau_{i}$ of each reflection is a function of the range cells, which is defined as

$$
\tau_{i}=2 \frac{R_{i}}{c}
$$

where $R_{i}$ represents the range of the $i$ th reflected scatterer. Substituting (20) into (19), we have

$$
\begin{aligned}
g\left(t_{r}\right) & =\sum_{i=0}^{N-1} \alpha_{i} \\
\cdot \exp & \left\{-j 4 \pi\left[\left(-\mu \frac{R_{i}}{c}-\frac{v_{0}}{c} f_{c}\right) t_{r}-f_{c} \frac{R_{i}}{c}+\mu \frac{R_{i}^{2}}{c^{2}}\right]\right\} .
\end{aligned}
$$

From the frequency term in (21), we can see that the Doppler component $f_{d 2}$ induced by radial motion is coupled with the term caused by the target ranges and induces a range migration in the HRRP. The superposition of the subtarget scatterers without Doppler shift is of the following form:

$$
\begin{aligned}
& \varepsilon\left(t_{r}\right) \\
& \quad=\sum_{i=0}^{N-1} \alpha_{i} \exp \left\{-j 4 \pi\left[-\mu \frac{R_{i}}{c} t_{r}-f_{c} \frac{R_{i}}{c}+\mu \frac{R_{i}^{2}}{c^{2}}\right]\right\} .
\end{aligned}
$$

Substituting (22) into (14) yields

$$
z\left(t_{r}\right)=\exp \left\{j 2 \pi \mu \frac{v_{0}}{c} t_{r}^{2}\right\} \cdot \exp \left\{j 4 \pi \frac{v_{0}}{c} f_{c} t_{r}\right\} \cdot \varepsilon\left(t_{r}\right) .
$$

From (18), (21), and (23) we can conclude that the there are two components caused by Doppler distortion: component $f_{d 1}$ results in the dispersion of the subtargets and component $f_{d 2}$ contributes to the range migration of the subtargets. Substituting (22) into (11), we get the ideal HRRP with the form of

$$
U(\omega)=\int_{t_{r}=2 R_{\min } / c}^{t_{r}=2 R_{\max } / c} \varepsilon\left(t_{r}\right) e^{-j \omega t_{r}} d t_{r} .
$$

The spectral peak in frequency domain corresponds to the range bins of each scatterer.

\section{Compensation of Doppler Distortion and Velocity Estimation Using Modified FrFT}

Subtargets dispersion and range migration caused by high speed target motion prevent the achievement of HRRP in a wide band radar with LFM transmitting. Hence, feature extraction from the HRRP becomes impractical without the compensation of the Doppler distortion. The approach of FrFT is proposed in this paper to compensate the Doppler distortion. The match effect of the FrFT is appropriate for the Doppler compensation of high speed moving target with uniform motion in a straight line.

3.1. Performance Analysis of FrFT. Fractional Fourier transform (FrFT) is now widely used for the analysis of signals with the waveform of LFM. The return of the LFM signal has firstly been dechirped by SP. Then, FrFT is used to compensate for the remaining frequency modulated residuals caused by target motion. Target echoes with different chirp rates in formula (23) can be accumulated by exploiting FrFT with different rotation angles. Hence, FrFT can be regarded as 
a rotation transform in the time-frequency coordinates. The FrFT on the output signal of SP is defined as $[32,33]$ where $p$ is the transform order. The transformation kernel $K_{p}\left(t_{r}, \omega\right)$ is defined as

$$
Z(\omega)=\int_{t_{r}=-2 R_{\min } / c}^{t_{r}=2 R_{\max } / c} z\left(t_{r}\right) K_{p}\left(t_{r}, \omega\right) d t_{r}
$$

$$
K_{p}\left(t_{r}, \omega\right)= \begin{cases}\sqrt{\frac{1-j \cot \beta}{2 \pi}} \exp \left\{j\left[\frac{t_{r}^{2}+\omega^{2}}{2} \cot \beta-\omega t_{r} \csc \beta\right]\right\}, & \beta \neq l \pi \\ \delta\left(t_{r}-\omega\right), & \beta=2 l \pi \\ \delta\left(t_{r}+\omega\right), & \beta=(2 l \pm 1) \pi,\end{cases}
$$

where $l$ is an integer, $\beta$ the rotation angle, $\cot [\cdot]$ the cotangent operation, and $\csc [\cdot]$ the cosecant operation. Substituting formula (26) into formula (25), we get

$$
\begin{gathered}
Z(\omega)=\sqrt{\frac{1-j \cot \beta}{2 \pi}} \exp \left\{j \frac{\omega^{2}}{2} \cot \beta\right\} \\
\cdot \int_{t_{r}=2 R_{\min } / c}^{t_{r}=2 R_{\max } / c} z\left(t_{r}\right) \exp \left\{j \frac{\cot \beta}{2} t_{r}^{2}\right\} \\
\cdot \exp \left\{-j \cdot \csc \beta \cdot \omega t_{r}\right\} d t_{r} .
\end{gathered}
$$

If the Doppler effect of a moving target matches the best transform angle, the echo signals can be well accumulated to be single frequencies. By letting

$$
q\left(t_{r}\right)=z\left(t_{r}\right) \exp \left\{j \frac{\cot \beta}{2} t_{r}^{2}\right\} \exp \left\{-j \csc \beta \cdot \omega t_{r}\right\}
$$

formula (27) becomes

$$
\begin{aligned}
Z(\omega)= & \sqrt{\frac{1-j \cot \beta}{2 \pi}} \exp \left\{j \frac{\omega^{2}}{2} \cot \beta\right\} \\
& \cdot \int_{t_{r}=2 R_{\min } / c}^{t_{r}=2 R_{\max } / c} q\left(t_{r}\right) d t_{r} .
\end{aligned}
$$

The item $\exp \left\{j \cot \beta \cdot t_{r}^{2} / 2\right\}$ in (28) shows the rotation function of the FrFT. The slope rate of the kernel function $\exp \left\{j(\cot \beta / 2) t_{r}^{2}\right\}$ changes with the value of $\beta$. When the slope rate of the kernel function is matched with the signal, there will be a peak after the FrFT. The FrFT corresponds to the identity operator when $\beta=0$ or a multiple of $2 \pi$. For $\beta=\pi / 2$, the FrFT takes the standard Fourier transform [28].

From (23) and (28), we get

$$
\begin{aligned}
q\left(t_{r}\right)= & \exp \left\{j \frac{\cot \beta}{2} t_{r}^{2}\right\} \exp \left\{-j \csc \beta \cdot \omega t_{r}\right\} \\
& \cdot \exp \left\{j 2 \pi \mu \frac{v_{0}}{c} t_{r}^{2}\right\} \exp \left\{j 4 \pi \frac{v_{0}}{c} f_{c} t_{r}\right\} \cdot \varepsilon\left(t_{r}\right) .
\end{aligned}
$$

The compensation of the target dispersion term can be defined as

$$
w\left(t_{r}\right)=\exp \left\{j \frac{\cot \beta}{2} t_{r}^{2}\right\} \exp \left\{j 2 \pi \mu \frac{v_{0}}{c} t_{r}^{2}\right\} .
$$

From (31), we can conclude that equation $\left|w\left(t_{r}\right)\right|^{2}=1$ is established if

$$
-\frac{\cot \beta}{2}=2 \pi \mu \frac{v_{0}}{c}
$$

where $|\cdot|$ denotes modulus operation. Hence, the velocity of the group target can be attained by calculating the following formula:

$$
v_{0}=-\frac{c \cdot \cot \beta}{4 \mu \pi}
$$

On the other hand, the range of parameter $\beta$ can be defined by setting the value of the velocity $v_{0}$ :

$$
\beta=\operatorname{arccot}\left(-4 \pi \mu \frac{v_{0}}{c}\right) .
$$

Though the compensation of the target dispersion component is accomplished, the range migration component remains:

$$
q\left(t_{r}\right)=\exp \left\{-j \csc \beta \cdot \omega t_{r}\right\} \exp \left\{j 4 \pi \frac{v_{0}}{c} f_{c} t_{r}\right\} \varepsilon\left(t_{r}\right) .
$$

It should be noted that the FrFT algorithm can not compensate both components of Doppler distortion at the same time.

3.2. Achieving HRRP Using Modified FrFT. To compensate the first item and the second item of $z\left(t_{r}\right)$ in (23) simultaneously, we define a modified version of FrFT. The new kernel function is defined as

$$
K_{M}\left(t_{r}\right)=\exp \left\{-j 2 \pi \mu \frac{v}{c} t_{r}^{2}\right\} \exp \left\{-j 4 \pi \frac{v}{c} f_{c} t_{r}\right\},
$$

where $v$ is the variable for velocity searching. The principle of defining such a kernel function is based on the fact that the target radial velocity is the only unknown factor in (23). The chirp rate of the transmitted signal, the signal window for analysis, the transmitted carrier frequency, and the pulse width are all a priori information in our radar system. The search of $v$ is viable in practical applications because the range of a target's velocity is a priori knowledge. Target dispersion and range migration both can be compensated by the utilization of (36) simultaneously. Hence, variable $v$ is not 
a simplified substitution to $\beta$ in (26). We can also find that item $\exp \left\{j(\cot \beta / 2) t_{r}^{2}\right\}$ in (28) has the function of rotation, which is similar to the item $\exp \left\{-j 2 \pi \mu(v / c) t_{r}^{2}\right\}$ in (36). Since the function of rotation is part of FrFT, we term the transform using formula (36) as modified FrFT. The transformation of the modified FrFT is of the following form:

$$
Z^{\prime}(\omega)=\int_{t_{r}=-2 R_{\min } / c}^{t_{r}=2 R_{\max } / c} z\left(t_{r}\right) K_{M}\left(t_{r}\right) \exp \left\{-j \omega t_{r}\right\} d t_{r} .
$$

Considering $v$ as another dimension as frequency $\omega$, (37) can be expressed as

$$
\begin{aligned}
& \chi(\omega, v) \\
& \quad=\int_{t_{r}=-2 R_{\min } / c}^{t_{r}=2 R_{\max } / c} z\left(t_{r}\right) K_{M}\left(v, t_{r}\right) \exp \left\{-j \omega t_{r}\right\} d t_{r} .
\end{aligned}
$$

By defining

$$
\begin{aligned}
q^{\prime}\left(t_{r}\right)= & z\left(t_{r}\right) K_{M}\left(v, t_{r}\right) \\
= & \exp \left\{j 2 \pi \mu \frac{v_{0}}{c} t_{r}^{2}\right\} \exp \left\{j 4 \pi \frac{v_{0}}{c} f_{c} t_{r}\right\} \cdot \varepsilon\left(t_{r}\right) \\
& \cdot \exp \left\{-j 2 \pi \mu \frac{v}{c} t_{r}^{2}\right\} \exp \left\{-j 4 \pi \frac{v}{c} f_{c} t_{r}\right\} .
\end{aligned}
$$

Equation (38) can be rewritten as

$$
\chi(\omega, v)=\int_{t_{r}=-2 R_{\min } / c}^{t_{r}=2 R_{\max } / c} q^{\prime}\left(t_{r}\right) \exp \left\{-j \omega t_{r}\right\} d t_{r} .
$$

According to (21) and (24), $\omega$ is in proportion to the ranges of the subtargets. Hence, formula (40) can be expressed as

$$
\chi(R, v) \propto \chi(\omega, v)
$$

where $R$ represents the range variable and $R \in\left[R_{\min }, R_{\max }\right]$. The plane calculated from (41) is called range/velocity plane. The modulus of the range/velocity plane is calculated as follows:

$$
|\chi(R, v)|^{2}=\chi(R, v) \chi^{*}(R, v) .
$$

$|\cdot|$ represents the modulus calculation of the range/velocity plane. If noise is considered in the above analysis, the range/ Doppler plane of (42) can be written as

$$
|\chi(R, v)|^{2}=\chi(R, v) \chi^{*}(R, v)+\sigma^{2}
$$

where $\sigma^{2}$ is the variance of the noise $n(t)$ in the receiver. With the variation of $v$ and $R$ (or $\omega$ ), peak will occur in the twodimensional plane if the velocity and range bin of a scatterer are matched. The corresponding range bin and velocity can be calculated for each scatterer of the subtarget. For each resolving scatterer, there is a local maximum value in the range/velocity plane. Since all the subtargets of a group target fly at the same speed, the velocity of the group target can be estimated by searching the global maximum value on the two-dimensional plane. The range of velocity searching can be defined as follows:

$$
v=v_{\min }: \Delta v: v_{\max } \cdot
$$

$v_{\min }$ and $v_{\max }$ are the minimum and maximum velocity set according to a priori information, and $\Delta v$ is the step size of the velocity for searching. The so called a priori information refers to some general knowledge, such as the velocity of a target. For instance, we can define that the searching scope of the velocity of a target is limited in the scope of $0-700 \mathrm{~m} / \mathrm{s}$. Though the estimated velocity will be more accurate if the step size is smaller, the computational complexity increases significantly. Compromise must be made between accuracy and computational load. From $(39),|\chi(R, v)|$ reaches its maximum values in velocity dimension if the velocity of the target is matched:

$$
v=v_{0}
$$

The LFM scatterers in linear frequency modulation will be changed into a series of single frequencies without target dispersion and range migration:

$$
q^{\prime}\left(t_{r}\right)=\varepsilon\left(t_{r}\right)
$$

The HRRP can be achieved by frequency transformation of (46), which has been shown in (22) and (24). The HRRP of a group target with speed $v_{0}$ is a slice along the velocity dimension in the range/velocity plane, which can be expressed as

$$
P_{\mathrm{HRRP}}=\left|\chi\left(R, v_{0}\right)\right|
$$

If the velocity of a group target is estimated, HRRP without distortion can be achieved. The process of velocity estimation and HRRP achievement of a group target can be summarized as follows:

(1) SP is performed within the receiving signal window.

(2) The two-dimensional range/velocity plane is calculated using modified FrFT (formula (38)).

(3) Velocity of the group target is determined by searching the maximum value on the plane.

(4) HRRP can be achieved by extracting the slice from the two-dimensional plane after the compensation of the target velocity (formula (24)).

\section{Range Superresolution Profile Construction Using MUSIC}

In order to extract more features from the HRRP, MUSIC algorithm with superresolution capability is adopted to process the signal in (46), which is the output of the modified FrFT. MUSIC estimates frequencies in (46) by performing eigendecomposition on the covariance matrix of $q^{\prime}\left(t_{r}\right)$ after the velocity of the group target has been compensated. 
Defining $\mathbf{V}$ as a vector of $M$ samples $(M>N)$ from $q^{\prime}\left(t_{r}\right)$, we get [34]

$$
\begin{gathered}
{\left[\begin{array}{c}
\mathbf{V}(0) \\
\mathbf{V}(1) \\
\vdots \\
\mathbf{V}(M-1)
\end{array}\right]} \\
=\left[\begin{array}{cccc}
q^{\prime}(0) & q^{\prime}(-1) & \cdots & q^{\prime}(-M+1) \\
q^{\prime}(1) & q^{\prime}(0) & \cdots & q^{\prime}(-M+2) \\
\vdots & \vdots & \ddots & \vdots \\
q^{\prime}(M-1) & q^{\prime}(M) & \cdots & q^{\prime}(0)
\end{array}\right] .
\end{gathered}
$$

Considering the data model of MUSIC, the $M \times M$ covariance matrix in the expectation form is

$$
\mathbf{R}=E\left[\mathbf{V V}^{H}\right]=\mathbf{A} E\left[\mathbf{S S ^ { H }}\right] \mathbf{A}^{H}+\sigma^{2} \mathbf{I},
$$

where $E[\cdot]$ is the expectation operator, $\mathbf{I}$ is the $M \times M$ identity matrix, and $\mathbf{A}$ is the $M \times N$ Vandermonde matrix of the signal frequencies. $\omega_{i}$ denotes the $i$ th frequency and

$$
a\left(\omega_{i}\right)=\left[\begin{array}{llll}
1 & e^{-j \omega_{i}} & \cdots & e^{-j(M-1) \omega_{i}}
\end{array}\right]^{T},
$$

where $a\left(\omega_{i}\right)$ is the $i$ th column of $\mathbf{A}$, and $\mathbf{A}$ can be expressed as

$$
\mathbf{A}=\left[a\left(\omega_{0}\right), a\left(\omega_{1}\right), \ldots, a\left(\omega_{i}\right), \ldots, a\left(\omega_{N}\right)\right] .
$$

$\boldsymbol{S}$ in (49) can be expressed as

$$
\mathbf{S}=\left[s_{0}, s_{1}, \ldots, s_{i}, \ldots, s_{N-1}\right]^{T},
$$

where $s_{i}$ is the $i$ th signal with $M$ samples, which is defined in formula (4). Let $\lambda_{i}$ be the eigenvalue and $\mathbf{G}_{i}$ be the eigenvector of covariance matrix $\mathbf{R}$. The eigenvectors associated with the $N$ maximum eigenvalues are defined as the signal subspace $\mathbf{U}_{s}$, and the other eigenvectors are defined as the noise subspace, $\mathbf{U}_{n}$. The eigendecomposition of $\mathbf{R}$ is as follows:

$$
\mathbf{R}=\mathbf{U}_{s} \Sigma_{s} \mathbf{U}_{s}^{H}+\mathbf{U}_{n} \Sigma_{n} \mathbf{U}_{n}^{H},
$$

where $[\cdot]^{H}$ denotes Hermitian transposition, and

$$
\begin{aligned}
\mathbf{U}_{s} & =\left(\mathbf{G}_{0}, \ldots, \mathbf{G}_{N-1}\right) \\
\mathbf{U}_{n} & =\left(\mathbf{G}_{N}, \ldots, \mathbf{G}_{M-1}\right) \\
\Sigma_{s} & =\operatorname{diag}\left(\lambda_{0}, \ldots, \lambda_{N-1}\right) \\
\Sigma_{n} & =\operatorname{diag}\left(\lambda_{N}, \ldots, \lambda_{M-1}\right) .
\end{aligned}
$$

From the orthogonality of the signal and noise subspaces, find the peaks in the estimator function

$$
P_{\text {MUSIC }}=\frac{1}{A^{H} \mathbf{U}_{n} \mathbf{U}_{n} A} .
$$

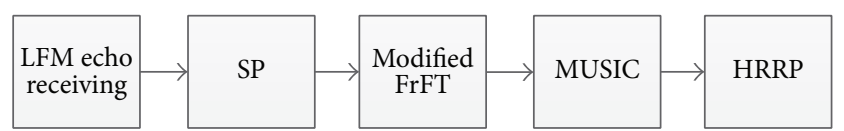

Figure 3: Processing chain of the proposed approach.

The peaks estimated from (55) correspond to the range bins of the subtargets of the group target.

As shown in Figure 3, processing approach consisting of SP, modified FrFT, and MUSIC algorithm is used to construct the processing chain for radar systems with wide band LFM pulse transmission. The difference between the proposed approach and the methods in Figures 1 and 2 lies in the utilization of modified FrFT. The variations of the signals in time-frequency domain are shown in Figure 4 to express the effect of modified FrFT. The processing chain can be summarized as follows:

(1) Reception of the wide band LFM signal (formula (7)): the time-frequency characteristic of the received scatterers is shown in Figure 4(b).

(2) SP is used in the receiving channel for bandwidth compression, transforming the reflections of the LFM waveform into signals with Doppler distortion (formula (10). The sketch of the time-frequency spectrum is shown in Figure 4(c)).

(3) Modified FrFT is utilized to compensate the Doppler distortion. The received signal is transformed into superposition of single frequencies without distortion (formula (46). The sketch of the time-frequency spectrum is shown in Figure 4(d)).

(4) HRRP is obtained by calculating the spectrum of the MUSIC algorithm (formula (55)).

\section{Numerical Simulations}

To evaluate the performance of the modified FrFT, a missile with multiple warheads flying in close formation was emulated. Three algorithms were selected for comparison study in the numerical simulations, including the conventional method of SP, the method of SP and FrFT, and the combined method using SP, FrFT, and MUSIC algorithms. We term the three algorithms as follows:

Method I: the method of SP (realized by FFT).

Method II: the method of SP + modified FrFT.

Method III: the method of SP + modified FrFT + MUSIC.

5.1. Compensation of Doppler Distortion Using Modified FrFT. Comparison study was carried out between method I and method II to observe the compensating performance for Doppler distortion using modified FrFT. As shown in Table 1, three warheads with different range cells were simulated.

The conditions of numerical simulations are as follows:

(1) Bandwidth: $200 \mathrm{MHz}$.

(2) Sampling rate: $400 \mathrm{MHz}$. 


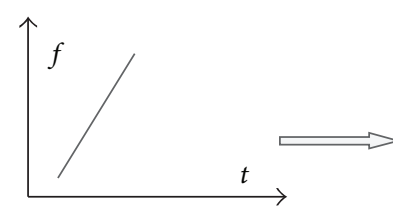

(a)

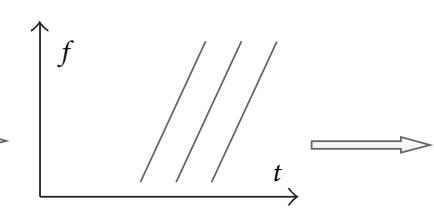

(b)

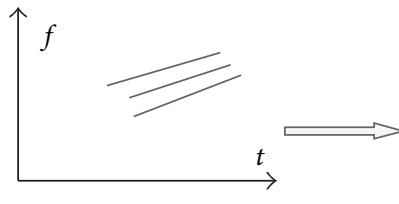

(c)

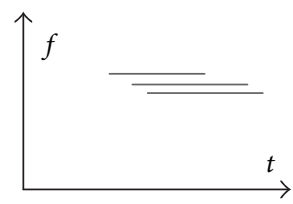

(d)

FIGURE 4: Characteristic changes in time-frequency domain in different phase of the proposed processing chain. (a) Transmitted signal. (b) Received scatterers. (c) Scatterers after SP. (d) Scatterers after FrFT.

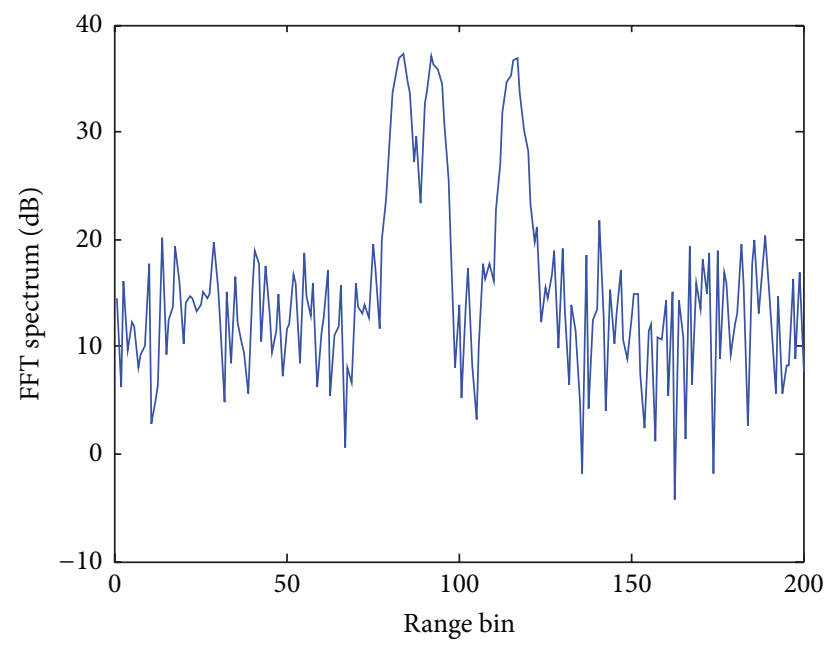

FIGURE 5: The HRRP results using method I.

TABLE 1: The range cells of the subtargets added in simulation.

\begin{tabular}{lccc}
\hline Subtarget & Warhead 1 & Warhead 2 & Warhead 3 \\
\hline Range cell & 97 & 107 & 130 \\
\hline
\end{tabular}

(3) Signal-to-noise ratio: $24 \mathrm{~dB}$ (after SP).

(4) Target velocity: $5000 \mathrm{~m} / \mathrm{s}$.

(5) Pulse width: $30 \mu \mathrm{s}$.

(6) Chebyshev weighting of $30 \mathrm{~dB}$ being selected as the window added to the receiving data.

(7) The range for observation being $150 \mathrm{~m}$ (200 samples), and the signal window for SP being selected as the pulse width.

(8) The step size for velocity searching being set to $20 \mathrm{~m} / \mathrm{s}$.

Figure 5 clearly demonstrates that two of our three subtargets are too close to be resolved by conventional method I. There is a range shift for all three subtargets, which is far from the true value in Table 1. Figure 6 illustrates the range/ velocity plane using the modified FrFT. From Figure 6(b), the contour plot of Figure 6(a), it can be found that scatterers with better resolution can be achieved in the range domain as the compensating velocity is approaching the real value. We determine velocity of the group target by searching the maximum peak value on the two-dimensional range/velocity plane. The estimated velocity was $4980 \mathrm{~m} / \mathrm{s}$, with an error of $20 \mathrm{~m} / \mathrm{s}$. Figure 7 then illustrates the estimated HRRP
TABLE 2: The range cells of the multiple warheads added in simulation.

\begin{tabular}{lcccc}
\hline Subtarget & Warhead 1 & Warhead 2 & Warhead 3 & Warhead 4 \\
\hline Range cell & 122 & 126 & 130 & 136 \\
\hline
\end{tabular}

obtained by method II. Note that method II with Doppler compensation clearly resolves the three subtargets and accurately locates their range positions. The range shift was successfully compensated and the positions of the scatterers were correctly located.

5.2. The Performance of Velocity Estimation Using Modified FrFT. Monte Carlo simulation of 100 times was used to evaluate the performance of target velocity estimation using method II. The simulation conditions were the same as those used in Section 5.1. The estimated mean value and standard variance of the target velocity in different SNR conditions are given in Figures 8 and 9, respectively. Figure 8 shows that the estimated mean value approaches its true value $5000 \mathrm{~m} / \mathrm{s}$ as SNR increases. From Figure 9, we can see that the standard variance of the velocity tails off with the increase of the SNR. In other words, the deviation of the measurement from its true value is diminishing as SNR increases.

5.3. Performance Comparison between Method II and Method III. The difference of resolution between method II and method III is investigated in this simulation. The simulation conditions are as follows:

(1) Transmitting signal pulse width: $50 \mu \mathrm{s}$.

(2) The bandwidth of the signal: $600 \mathrm{MHz}$.

(3) The sampling frequency: $1200 \mathrm{MHz}$.

(4) SNR: $26 \mathrm{~dB}$ (after SP).

(5) Target velocity: $3600 \mathrm{~m} / \mathrm{s}$.

Other conditions are the same as those in Section 5.1. The range cells of the subtargets (warheads) added in the simulation are listed in Table 2.

Two groups of simulation with different SNR were carried out to test the performance of method II and method III. The target velocity was firstly estimated and compensated using modified FrFT when using method II and method III. When SNR was set to $30 \mathrm{~dB}$ and $25 \mathrm{~dB}$, the estimated values of the velocity were $3640 \mathrm{~m} / \mathrm{s}$ and $3680 \mathrm{~m} / \mathrm{s}$, respectively. The HRRP results of method II in different SNR conditions are 


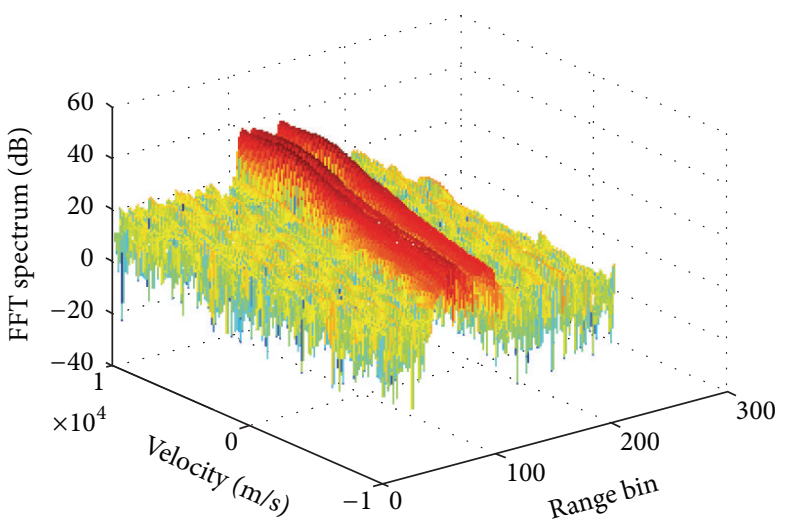

(a) Range/velocity plane using modified FrFT

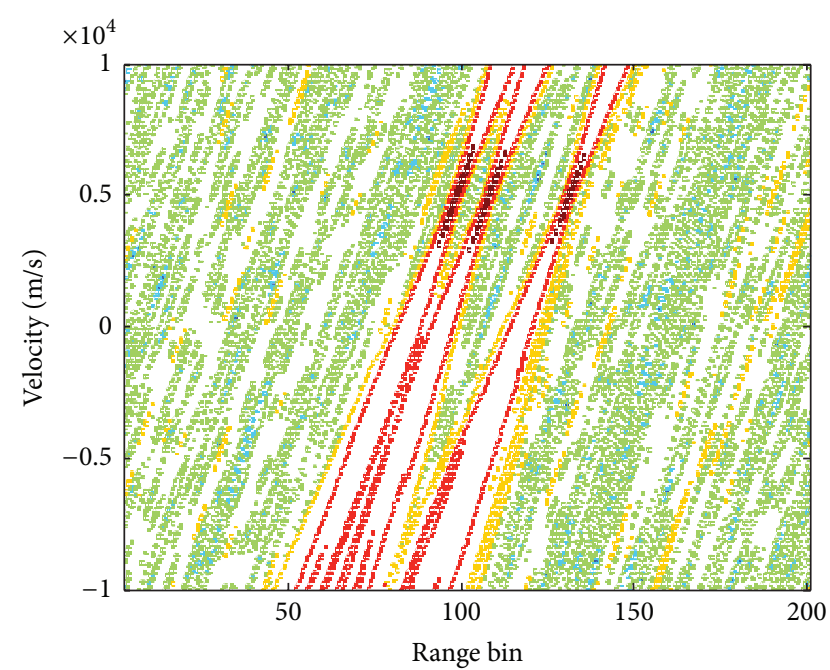

(b) The contour plot of (a)

FIGURE 6: Velocity searching using modified FrFT.

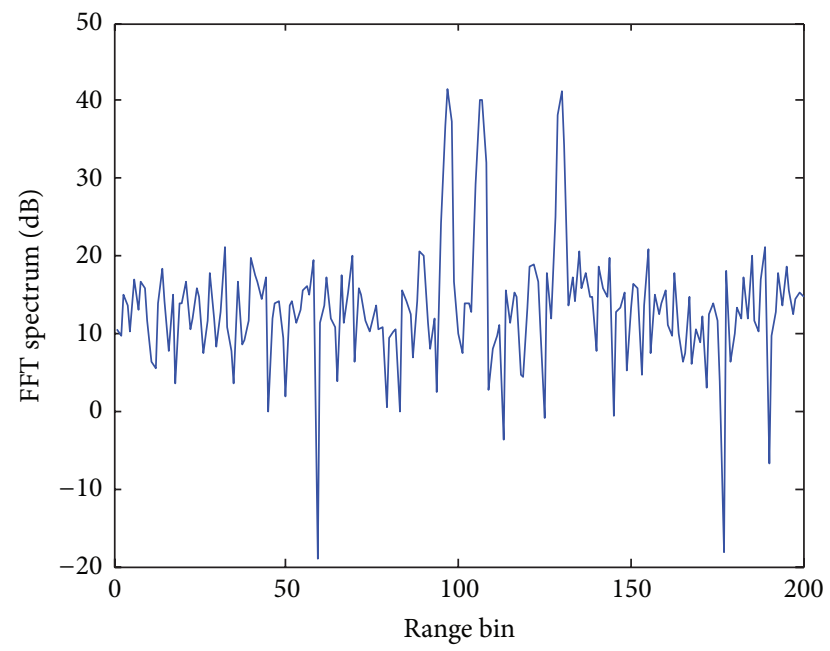

FIgURE 7: The HRRP results using method II.

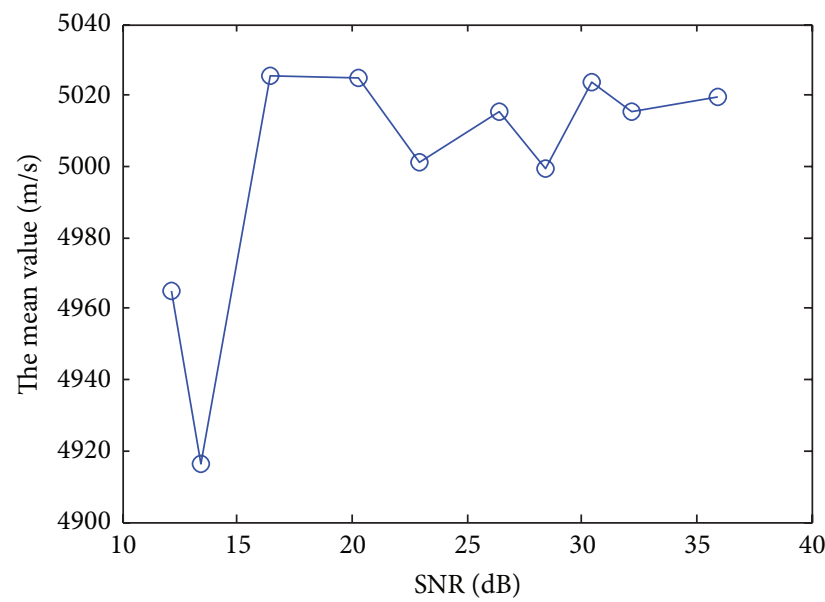

FIgURE 8: The mean value of the estimated velocity using modified FrFT.

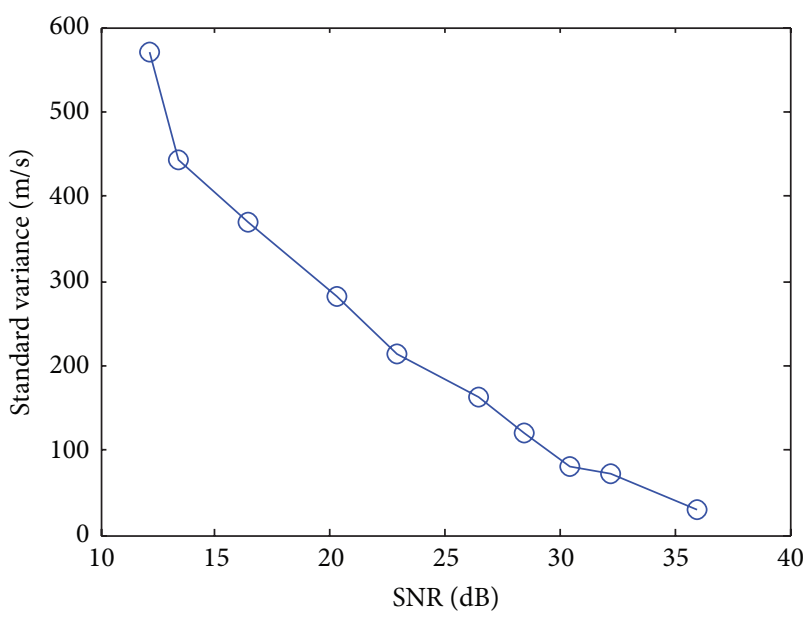

FIGURE 9: The standard variance of the estimated velocity using modified FrFT.

shown in Figures 10 and 11. Note that the subtargets 1-4 can not be identified. The results of method III in different SNR conditions are given in Figures 12 and 13, respectively. Four subtargets can be resolved by method III.

\section{Conclusions}

Superresolution of the high speed group target for wide band radar using LFM pulse is investigated in this paper. The target dispersion and range migration caused by the Doppler distortion in the wide band are compensated by the utilization of modified FrFT. Comparing with the conventional SP method, processing chain consisting of SP, modified FrFT and MUSIC algorithm have been adopted to achieve clearer discrimination between the subtargets. Exploiting the modified FrFT technique, the velocity of the group target can be estimated 


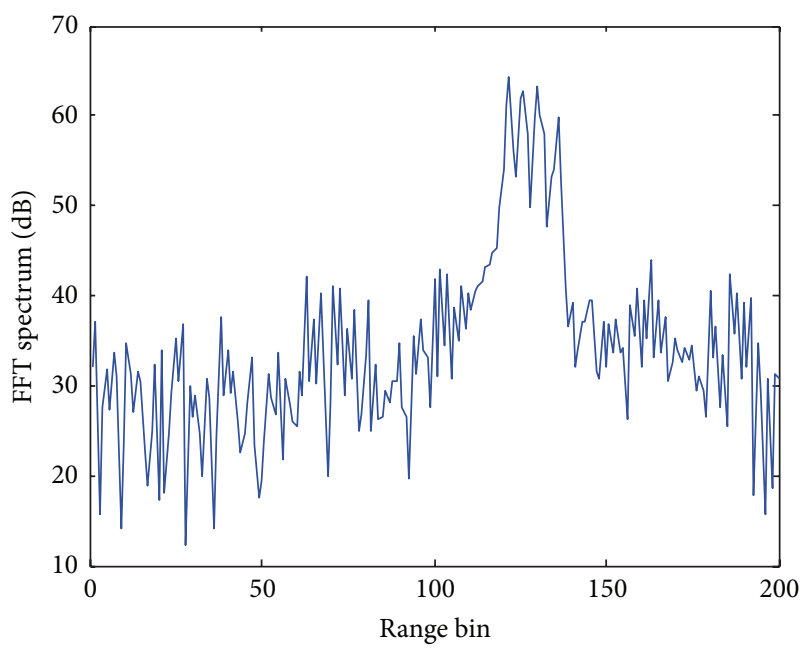

Figure 10: The results using method II (30 dB).

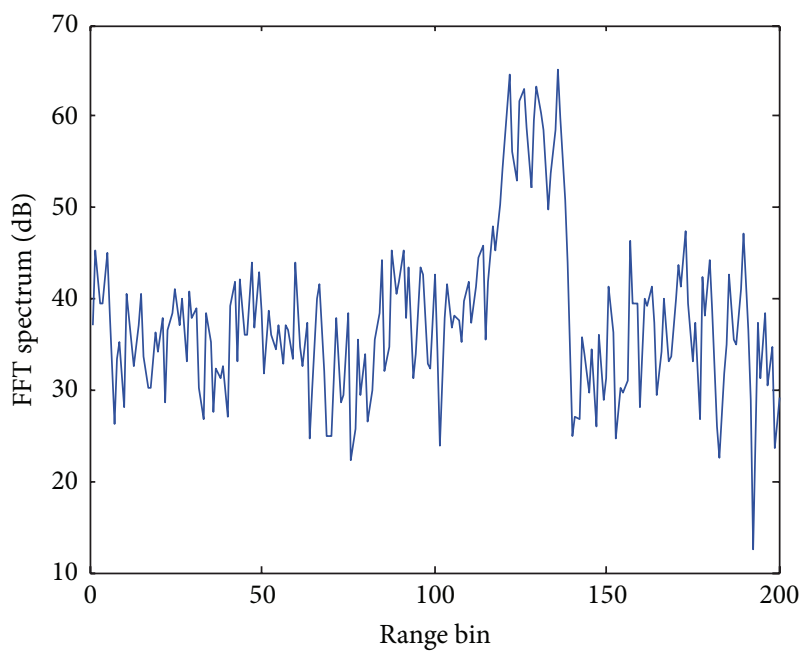

FIGURE 11: The results using method II ( $25 \mathrm{~dB})$.

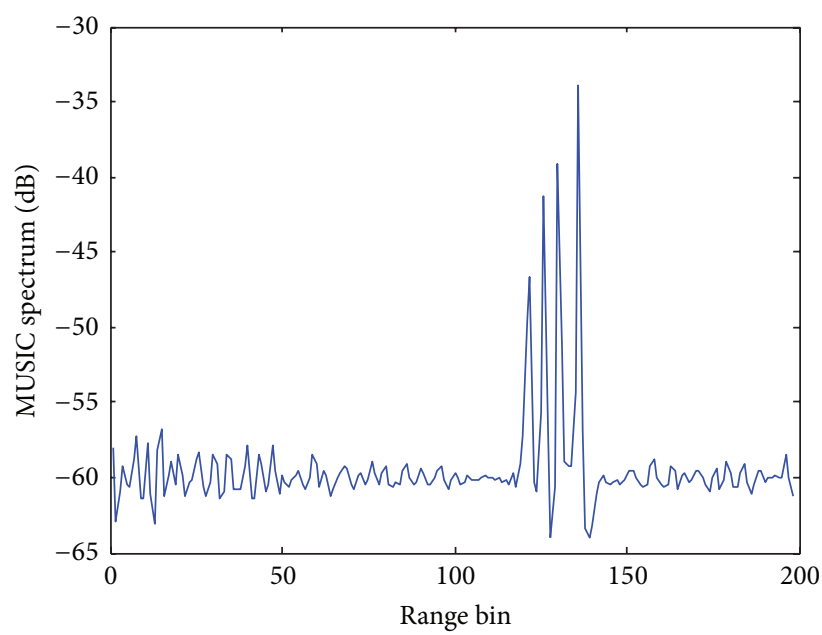

FIGURE 12: Superresolution results using method III (30 dB).

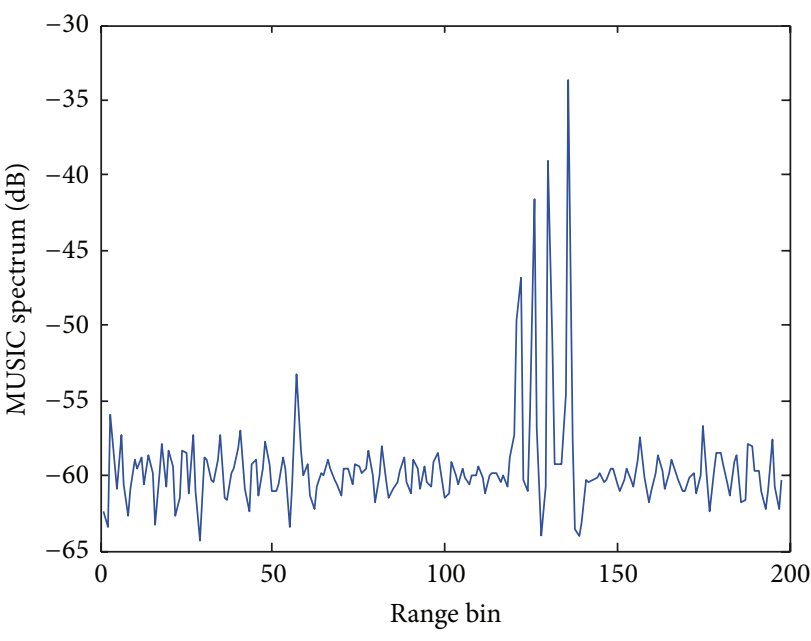

FIGURE 13: Superresolution results using method III (25 dB).

simultaneously with just one single pulse. The validity of the proposed approach is proven by computer simulation.

\section{Conflict of Interests}

The authors declare that there is no conflict of interests regarding the publication of this paper.

\section{Acknowledgments}

This work was partially supported by the Fundamental Research Funds for the Central Universities (Grant no. 2015B03014) and Natural Science Foundation of Jiangsu Province (Grant no. BK20151501).

\section{References}

[1] K. Granstrom and U. Orguner, "On spawning and combination of extended/group targets modeled with random matrices," IEEE Transactions on Signal Processing, vol. 61, no. 3, pp. 678692, 2013.

[2] G. Gao, "An improved scheme for target discrimination in highresolution SAR images," IEEE Transactions on Geoscience and Remote Sensing, vol. 49, no. 1, pp. 277-294, 2011.

[3] X. Bai, F. Zhou, M. Xing, and Z. Bao, "A novel method for imaging of group targets moving in a formation," IEEE Transactions on Geoscience and Remote Sensing, vol. 50, no. 1, pp. 221231, 2012.

[4] A. Gning, L. Mihaylova, S. Maskell, S. K. Pang, and S. Godsill, "Group object structure and state estimation with evolving networks and Monte Carlo methods," IEEE Transactions on Signal Processing, vol. 59, no. 4, pp. 1383-1396, 2011.

[5] S. P. Jacobs and J. A. O’Sullivan, "Automatic target recognition using sequences of high resolution radar range-profiles," IEEE Transactions on Aerospace and Electronic Systems, vol. 36, no. 2, pp. 364-381, 2000.

[6] S. K. Wong, "High range resolution profiles as motion-invariant features for moving ground targets identification in SAR-based automatic target recognition," IEEE Transactions on Aerospace and Electronic Systems, vol. 45, no. 3, pp. 1017-1039, 2009. 
[7] H.-J. Li and S.-H. Yang, "Using range profiles as feature vectors to identify aerospace objects," IEEE Transactions on Antennas and Propagation, vol. 41, no. 3, pp. 261-268, 1993.

[8] W. F. Gabriel, "Superresolution techniques in the range domain," in Proceedings of the IEEE International Radar Conference Digest, pp. 263-267, IEEE, Arlington, Va, USA, May 1990.

[9] C. Chen and Z. Xiaoling, "A new super-resolution 3D-SAR imaging method based on MUSIC algorithm," in Proceedings of the IEEE Radar Conference (RADAR '11), pp. 525-529, IEEE, Kansas City, Mo, USA, May 2011.

[10] M. Hurst and R. Mittra, "Scattering center analysis via Prony's method," IEEE Transactions on Antennas \& Propagation, vol. 35, no. 8, pp. 986-988, 1987.

[11] E. K. Walton, "Comparison of Fourier and maximum entropy techniques for high-resolution scattering studies," Radio Science, vol. 22, no. 3, pp. 350-356, 1987.

[12] R. L. Moses and J. W. Carl Jr., "Autoregressive modeling of radar data with application to target identification," in Proceedings of the IEEE National Radar Conference Digest, pp. 220-224, IEEE, Ann Arbor, Mich, USA, April 1988.

[13] Y. Chen and R. Berkowih, "Characteristics of autoregressive spectral estimation in range-Doppler imaging," in Proceedings of the 3rd IEEE National Radar Conference, pp. 118-122, Ann Arbor, Mich, USA, April 1988.

[14] K.-T. Kim, D.-K. Seo, and H.-T. Kim, "Efficient radar target recognition using the MUSIC algorithm and invariant features," IEEE Transactions on Antennas and Propagation, vol. 50, no. 3, pp. 325-337, 2002.

[15] Z. Bi, R. Wu, J. Li, and R. Williams, "Joint super-resolution moving target feature extraction and stationary clutter suppression," IEE Proceedings on Radar, Sonar \& Navigation, vol. 147, no. 1, pp. 23-29, 2000.

[16] S. D. Blunt, K. Gerlach, and T. Higgins, "Aspects of radar range super-resolution," in Proceedings of the IEEE National Radar Conference, pp. 683-687, IEEE, Boston, Mass, USA, April 2007.

[17] W. F. Gabriel, "Improved range superresolution via bandwidth extrapolation," in Proceedings of the IEEE National Radar Conference, pp. 123-127, IEEE, Lynnfield, Mass, USA, April 1993.

[18] T. H. Einstein, "Generation of high resolution radar range profiles and range profile autocorrelation functions using stepped frequency pulse trains," Project Report TT-54 (AD-A149242), MIT Lincoln Laboratory, Lexington, Mass, USA, 1984.

[19] N. Levanon, "Stepped-frequency pulse-train radar signal," IEE Proceedings: Radar, Sonar and Navigation, vol. 149, no. 6, pp. 297-309, 2002.

[20] Y. Liu, H. Meng, G. Li, and X. Wang, "Velocity estimation and range shift compensation for high range resolution profiling in stepped-frequency radar," IEEE Geoscience and Remote Sensing Letters, vol. 7, no. 4, pp. 791-795, 2010.

[21] T. J. Abatzoglou and G. O. Gheen, "Range, radial velocity, and acceleration MLE using radar LFM pulse train," IEEE Transactions on Aerospace and Electronic Systems, vol. 34, no. 4, pp. 1070-1084, 1998.

[22] Y. Liu, H. Meng, H. Zhang, and X. Wang, "Motion compensation of moving targets for high range resolution steppedfrequency radar," Sensors, vol. 8, no. 5, pp. 3429-3437, 2008.

[23] C. Fukushima and N. Hamada, "A study on stepped frequency radar by using intra-pulse phase coded modulation," in Proceedings of the World Congress on Engineering and Computer Science, pp. 22-24, San Francisco, USA, 2008.
[24] H.-Y. Chen, Y.-X. Liu, W.-D. Jiang, and G.-R. Guo, "A new approach for synthesizing the range profile of moving targets via stepped-frequency waveforms," IEEE Geoscience and Remote Sensing Letters, vol. 3, no. 3, pp. 406-409, 2006.

[25] W. J. Caputi, "Stretch: a time-transformation technique," IEEE Transactions on Aerospace and Electronic Systems, vol. 7, no. 2, pp. 269-278, 1971.

[26] J. Misiurewicz and K. Kulpa, "Stretch processing for masking effect removal in noise radar," IET Radar, Sonar \& Navigation, vol. 2, no. 4, pp. 274-283, 2008.

[27] L. Yeh, K. T. Wong, and H. S. Mir, "Viable/inviable polynomialphase modulations for 'stretch processing"' IEEE Transactions on Aerospace and Electronic Systems, vol. 48, no. 1, pp. 923-926, 2012.

[28] H. M. Ozaktas, O. Ankan, M. Alper Kutay, and G. Bozdagi, "Digital computation of the fractional fourier transform," IEEE Transactions on Signal Processing, vol. 44, no. 9, pp. 2141-2150, 1996.

[29] X. Chen, J. Guan, Y. He, and J. Zhang, "Detection of low observable moving target in sea clutter via fractal characteristics in fractional Fourier transform domain," IET Radar, Sonar and Navigation, vol. 7, no. 6, pp. 635-651, 2013.

[30] G.-C. Sun, M. Xing, X.-G. Xia, J. Yang, Y. Wu, and Z. Bao, “A unified focusing algorithm for several modes of SAR based on FrFT," IEEE Transactions on Geoscience and Remote Sensing, vol. 51, no. 5, pp. 3139-3155, 2013.

[31] K. Singh, R. Saxena, and S. Kumar, "Caputo-based fractional derivative in fractional fourier transform domain," IEEE Journal on Emerging and Selected Topics in Circuits and Systems, vol. 3, no. 3, pp. 330-337, 2013.

[32] G.-C. Sun, M. Xing, X.-G. Xia, J. Yang, Y. Wu, and Z. Bao, “A unified focusing algorithm for several modes of SAR based on FrFT," IEEE Transactions on Geoscience and Remote Sensing, vol. 51, no. 5, part 2, pp. 3139-3155, 2013.

[33] S.-C. Pei and J.-J. Ding, "Fractional Fourier transform, Wigner distribution, and filter design for stationary and nonstationary random processes," IEEE Transactions on Signal Processing, vol. 58, no. 8, pp. 4079-4092, 2010.

[34] R. O. Schmidt, "Multiple emitter location and signal parameter estimation," IEEE Transactions on Antennas and Propagation, vol. 34, no. 3, pp. 276-280, 1986. 


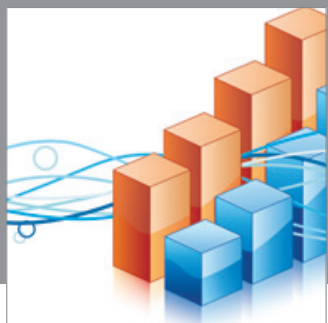

Advances in

Operations Research

mansans

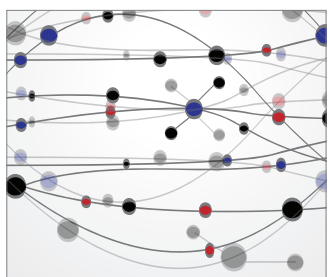

The Scientific World Journal
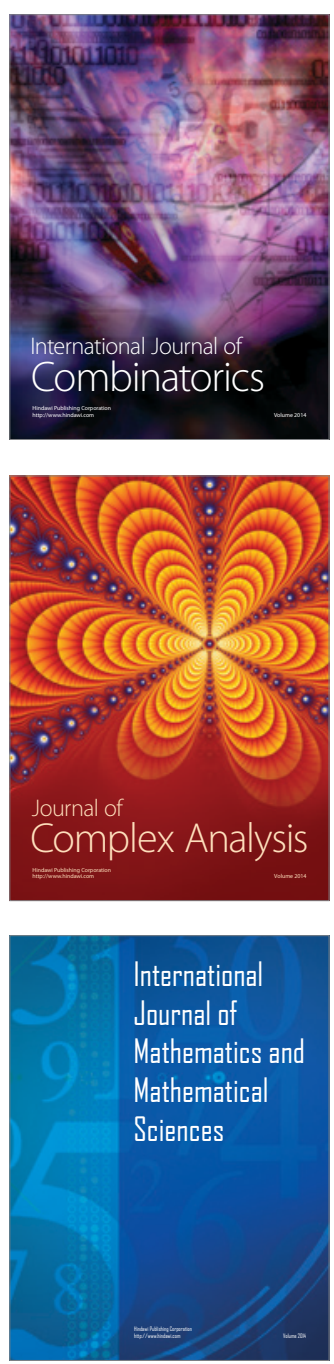
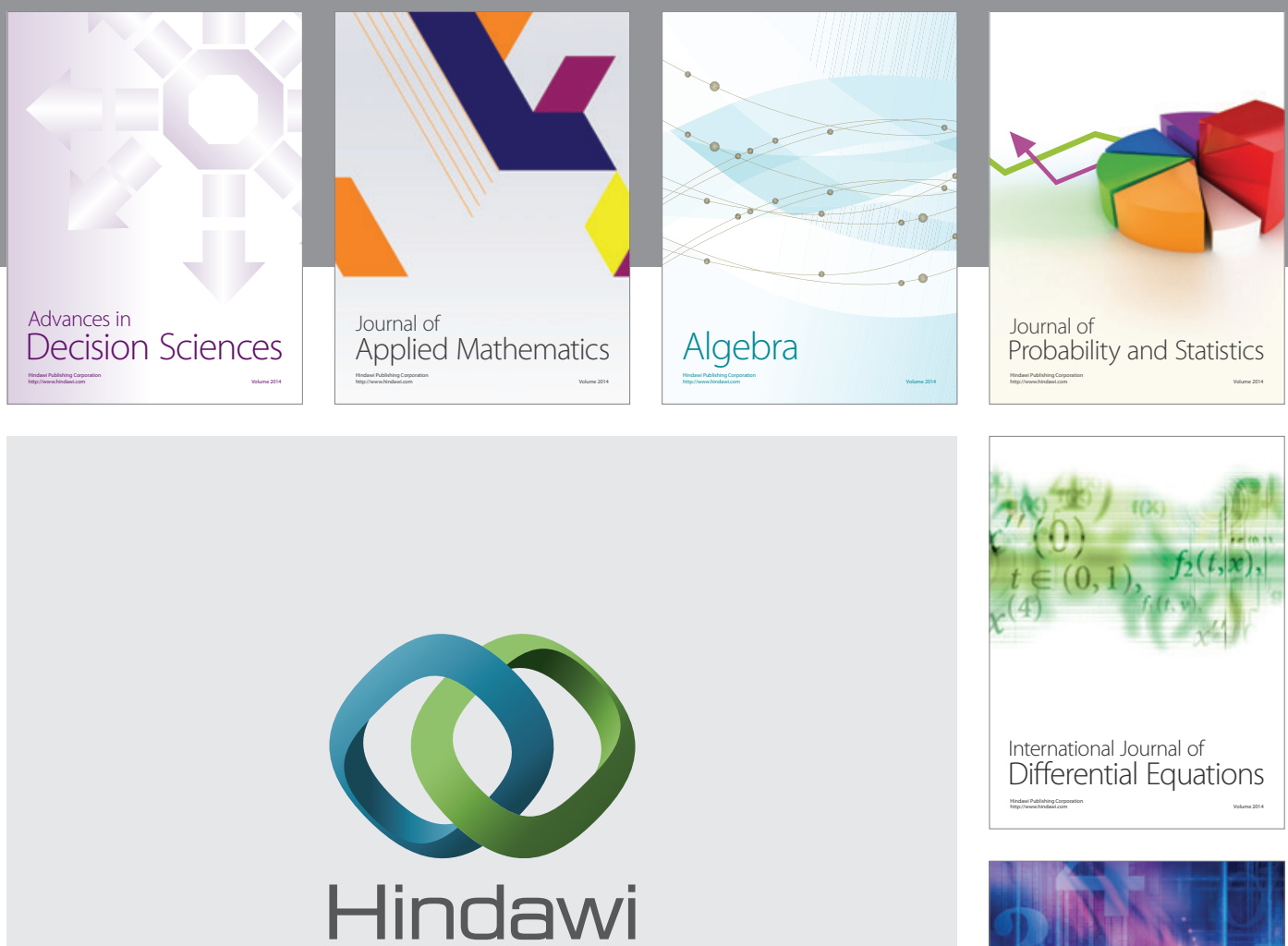

Submit your manuscripts at http://www.hindawi.com
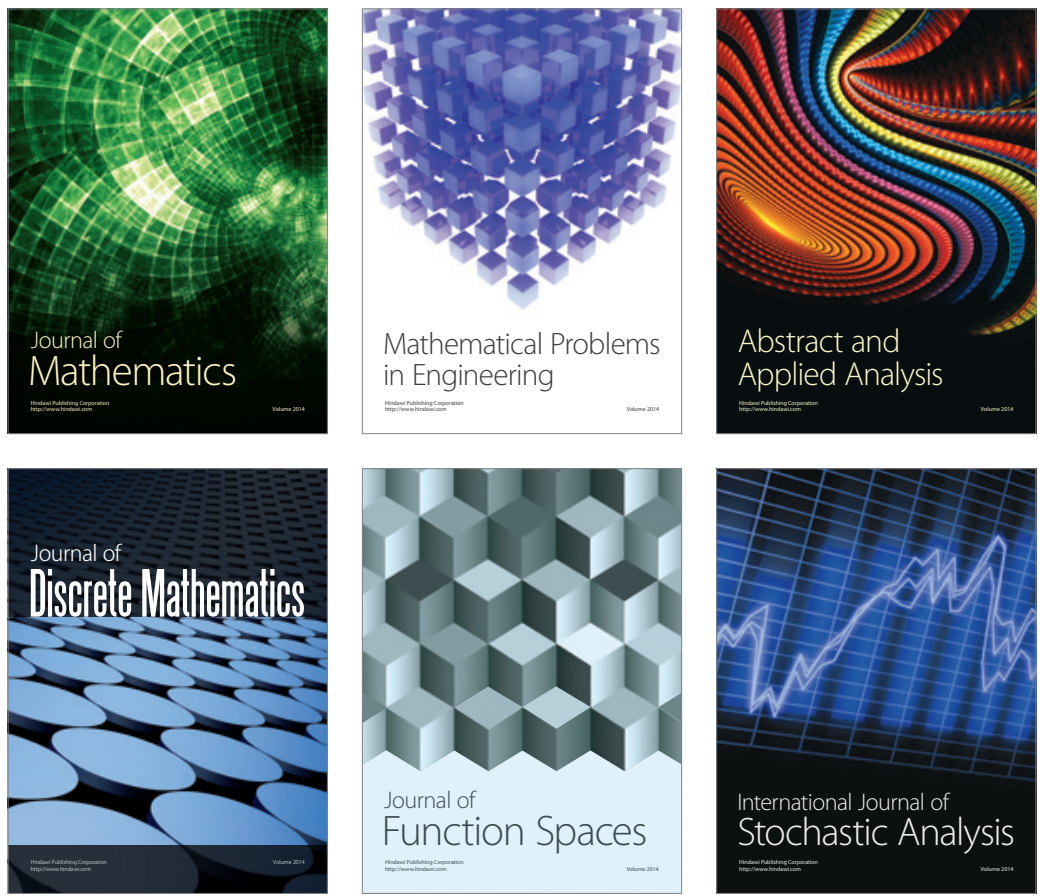

Journal of

Function Spaces

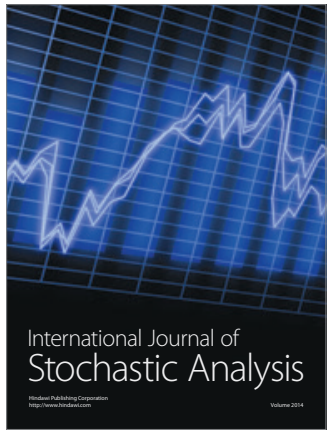

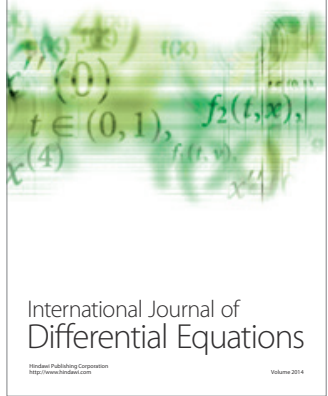
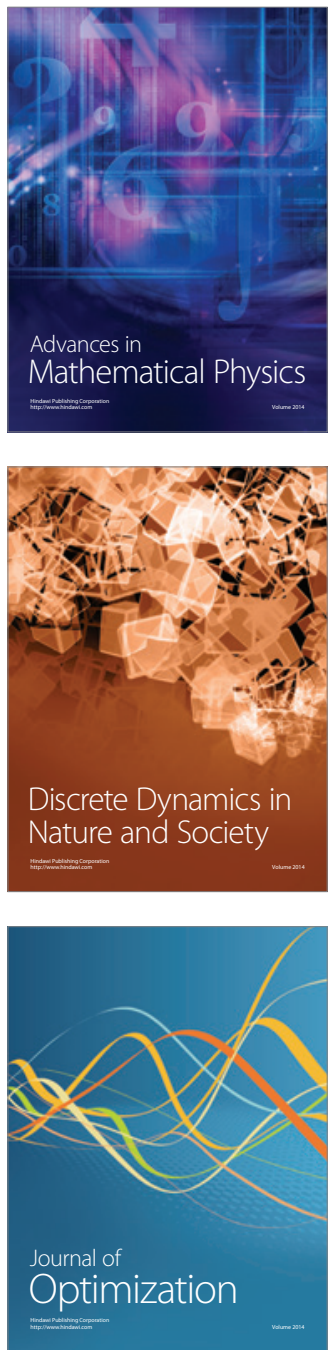